tion (cardiopulmonary resuscitation (CPR), thrombolytics, transfusion, intravenous medication).

Results: Category definitions of the developed DCS and the mean upper tolerance of $\mathrm{AE}$ risks were to develop a prognostic tool for real-time patient classification. Specific clinical variables (current vital signs, working diagnoses, co-morbidities, key laboratory results, functional status) were weighted and rank ordered by EPs for likelihood of predicting an $\mathrm{AE}$ within 72 hours of disposition. Details of these rankings will be presented.

Conclusion: The DCS, based on risk tolerance of AEs, allows conceptual classification of inpatients for safe disposition, allowing hospital capacity to be used for acutely ill or injured patients in a disaster.

\begin{tabular}{|c|l|c|}
\hline Rank & \multicolumn{1}{|c|}{ Definition } & $\begin{array}{c}\text { Mean Upper Risk } \\
\text { Tolerance of AE }\end{array}$ \\
\hline 1 & $\begin{array}{l}\text { Minimal Risk of } A E: \text { Suitable for } \\
\text { Discharge Home }\end{array}$ & $<4 \%$ \\
\hline 2 & $\begin{array}{l}\text { Low Risk of AE: Transfer to Low } \\
\text { Acuity Facility }\end{array}$ & $4-12 \%$ \\
\hline 3 & $\begin{array}{l}\text { Moderate Risk of AE: Transfer to } \\
\text { Facility to Moderate Capabilities }\end{array}$ & $13-33 \%$ \\
\hline 4 & $\begin{array}{l}\text { Significant Risk of AE: Transfer } \\
\text { to Major Acute Care Facility Only }\end{array}$ & $34-60 \%$ \\
\hline 5 & $\begin{array}{l}\text { High Risk of } A E: \text { Keep or } \\
\text { Transfer to ICU Setting Only }\end{array}$ & $>61 \%$ \\
\hline
\end{tabular}

Table 1-Category definitions of the Disposition of Classification System (DCS) developed by the expert panels $(\mathrm{AE}=$ adverse event; $\mathrm{ICU}=$ intensive care unit)

Keywords: adverse event; definition; disposition of classification system (DCS); cxpert panels; hospital; triage

Prehosp Disast Med 2005:20(2):s9-s10

\section{The Australian Capital Territory (ACT) General Practice Biothreat Preparedness Survey \\ A. Geysen; ${ }^{1}$ A. Herceg; ${ }^{2}$ C. Guest, ${ }^{3}$ R. Bialkowski ${ }^{1}$ \\ 1. ACT Division of General Practice, Australia \\ 2. Australasian Faculty of Public Health Medicine, Australia \\ 3. ACT Health, Australia}

In 2003, an outbreak of severe acute respiratory syndrome (SARS), a newly emerged infectious disease, caused a global public health emergency. In Australia, the response to SARS included specific guidelines for general practitioners $(\mathrm{GPs})$. These guidelines covered patient screening, infection control procedures, and specifications regarding equipment availability.

In late 2003 and early 2004, the ACT Division of General Practice and the ACT Health (the Territory's Health Authority) conducted two concurrent, anonymous, self-completion, postal surveys of all ACT GPs. The surveys were designed to identify knowledge, attitudes, and practices of GPs around SARS and biothreat preparedness. One survey asked individual GPs questions about: (1) how they gathered information on SARS in 2003; (2) how they prefer to receive information; (3) their current practices; and (4) how they perceived the threat of SARS and other infectious agents. The second survey asked practice princi- pals: (1) how they organized their general practice to respond to the SARS threat in 2003; (2) about any difficulties they had while implementing this response; (3) about the use of guidelines; and (4) about their current policies.

The response rate for the GP survey was $48 \%$ (184 of 381 ), and the response rate for the practice survey was $54 \%$ (74 of 136). Some issues raised by the survey will be discussed, as well as the ensuing recommendations. These issues included rapid communication with GPs in a public health emergency, application of guidelines in the general practice setting, occupational health and safety, continuing professional development, and GP involvement in planning for future outbreaks or public health emergencies. It is hoped that information obtained through these surveys will help the ACT and other parts of Australia improve future responses to emerging infectious disease threats.

Keywords: Australia; biothreat; general practitioners (GPs); guidelines; preparedness; severe acute respiratory syndrome (SARS); survey

Prebosp Disast Med 2005;20(2):s10

\section{A Public Health and Disaster Mitigation Model: Case Studies from Ecuador}

\section{Whiteford; G. Tobin; T. Mason}

Global Center for Disaster Management and Humanitarian Action,

Tampa, Florida USA

Based on five years of multidisciplinary health and social science research, the following model of Chronic Exposures Health and Hazards (CEHH) was developed. The CEHH model is a schematic presentation of multifactorial variables important to the design of health disaster mitigation strategies. Using this model, public health policies and programs can be designed to reduce health consequences of acute and chronic disasters. Disasters have long-term debilitating impacts on society, which can be manifested in higher levels of contagious/communicable diseases, increased vulnerability, early death, decreased social capital, and economic stress. Mitigating such impacts will limit human vulnerability and enhance various social, economic, and political characteristics, such as personal relationships, social contacts, shared interest groups, and other community-building and public health activities.

Since 1999, an international team composed of physicians, epidemiologists, public health professionals, and applied social scientists, collaborated to investigate the effects of ongoing exposure to volcanic risk. Research was undertaken in communities located on Mount Tungurahua, Ecuador, an active volcano that has been depositing ash over the surrounding landscape for the last five years. The research used a multi-dimensional, integrated model of relationships among different health outcome measures, as assessed through structured questionnaires and in-depth ethnographic studies of local residents, interviews with public health officials and political leaders, and evaluations of regional epidemiological and clinical records.

The results of the CEHH model suggest public health interventions in four areas: (1) integrated disaster planning to include a locally-based focus, extensive local community 
involvement, and inter-sectorial planning; (2) health communication and promotion policies that facilitate both vertical and horizontal communication and share information among government agencies, non-governmental organizations (NGOs), and local communities; (3) coordination of aid at local, regional, and global levels, among private and public inter-institutional relief groups, and identification of local contexts and international providers; and (4) creation of a global culture of health and hazard mitigation planning that is dynamic, ongoing, culturally appropriate, and that fosters comprehensive medical/public health training programs.

Keywords: communication; coordination; disaster; Ecuador; global culture; hazard; mitigation; model; planning; public health; training; volcano

Prehosp Disast Med 2005;20(2):s10-s11

\section{Arsenicosis: A Public Health Challenge \\ T.D. Banerjee \\ West Bengal Voluntary Health Association, India}

A large part of West Bengal, India and Bangladesh are located in the Gangetic delta. Nearly 76 blocks in nine districts of West Bengal and two-thirds of Bangladesh are contaminated by arsenic. New areas in other states of India have been identified as having arsenic contamination in ground water tables. Due to possible toxicity in humans from this contamination, corrective/preventative actions are needed on an emergency basis.

The World Health Organization (WHO) has identified the safe limit of arsenic contamination in drinking water as $0.01 \mathrm{mg} / \mathrm{l}$. The safe limit of arsenic contamination in drinking water tables has been identified by the Indian Government's Department of Public Health Engineering as $0.05 \mathrm{mg} / \mathrm{l}$. Nine districts are identified as being contaminated by arsenic, including the city of Kolkata. In total, approximately $38,677 \mathrm{~km}^{2}$ are affected, and place $13 \mathrm{mil}-$ lion people at risk for arsenic toxicity. A conservative estimate places six million people as consumers of arsenic-rich water, causing health problems in $>300,000$ people.

People living in arsenic-risk zones, who have not tested their drinking and cooking water using a shallow tube well (50-180 feet) are at the greatest risk for arsenicosis. Also at risk for developing arsenicosis are people in communities in which: (1) any kind of arsenic removal plants were developed, but the plant no longer is operative; (2) the people are not using filtered water from the plant for drinking or cooking; and (3) any kind of arsenicosis symptoms have been identified in even a single person. People at risk for arsenicosis include: (1) poor people whose nutritional intake capacity is low; and (2) those $<12$ years of age and the elderly, due to their low body defense mechanisms. In addition, women are more vulnerable than men because women usually spend more time in the home, and are more likely to drink contaminated water, while men usually consume water outside the home, leading to reduced toxicity.

There are several medical/research/engineering institutions in the state of West Bengal that are working on different aspects of arsenicosis, including treatment, research, and engineering. Their clinical inputs can be separated into four stages: (1) pre-clinical; (2) clinical; (3) complications; and (4) malignancy.

Arsenic is a major public health problem, especially in developing countries like India and Bangladesh. All of the stakeholders working in different dimensions must share information and work together for the public interest. The scientific information must be shared with the community, and some non-governmental organizations (NGOs) potentially could be partners.

Keywords: arsenic; Bangladesh; contamination; India; risk; treatment; water; West Bengal

Prebosp Disast Med 2005;20(2):s11

\section{Free Papers Theme 3: Children in Disasters}

\author{
Orthopedic Injuries in Children Following the Bam \\ Earthquake \\ H. Abolghasemi; M. Sabzecbian; M.H. Radfar; N. Jonaidi \\ Jafari; H. Ghasemzadeb \\ Iran
}

Introduction: Children are one of the most vulnerable age groups in natural disasters, in terms of both physical and psychological injuries. In this paper, pediatric orthopedic injuries following the earthquake in Bam, Iran, in December 2003 are assessed. Such an assessment helps determine the general preparedness necessary in a referral hospital that should admit pediatric patients in the aftermath of a natural disaster.

Methods: All patients < 16 years old who were referred from Bam to three hospitals in Tehran (Milad, Imam Hossein, and Baqiyatallah Hospital) within one week of the earthquake were included. Questionnaires containing items about demographic data, types of injury, and operative/non-operative treatment were completed by visiting the cases and using their medical records. Injuries of limbs and the pelvis are discussed as orthopedic injuries, and classified in two groups: (1) joint/bone injury; and (2) soft tissue injury. Patients also are classified in three age groups: (1) $<6$ years old; (2) 6-10 years old; and (3) 11-16 years old.

Results: A total of 119 patients $<16$ years old were admitted in the hospitals during the period of the study. Thirtythree patients $(27.7 \%)$ were $<6$ years old, $26(21.8 \%)$ were 6-10 years old, and $60(50.4 \%)$ were $11-16$ years old. Of these, nine patients (7.4\%) had only soft tissue injury, 25 patients (21.0\%) had only bone/joint injury, and 49 patients (41.1\%) had both kinds of injuries. In total, 83 patients $(69.7 \%)$ had orthopedic injury. Both kinds of injury were much more common in the lower extremities than the upper. Of patients who had orthopedic injuries, 52 (62.6\%) underwent surgery. The frequency of bone/joint injury and soft tissue injury was not significantly different among different age groups. Also, the need for surgery has not been correlated with a specific age group. The need for surgery was highest in patients suffering from both kinds of injury. Conclusion: Following an earthquake, most of the referred pediatric patients, regardless of age, suffer from orthopedic 